\title{
Effectiveness of an 810-nm Diode Laser in Addition to Non-surgical Periodontal Therapy in Patients With Chronic Periodontitis: A Randomized Single- Blind Clinical Trial
}

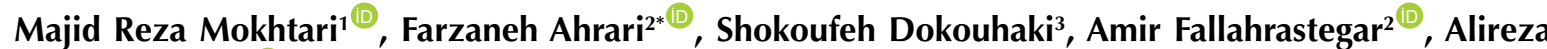 \\ Ghasemzadeh $^{3 * \mathbb{D}}$ \\ 'Department of Periodontontics, School of Dentistry, Mashhad University of Medical Sciences, Mashhad, Iran \\ ${ }^{2}$ Dental Research Center, School of Dentistry, Mashhad University of Medical Sciences, Mashhad, Iran \\ ${ }^{3}$ Student Research Committee, School of Dentistry, Mashhad University of Medical Sciences, Mashhad, Iran
}

\section{*Correspondence to Farzaneh Ahrari, \\ Dental Research Center, School of Dentistry, Mashhad University of Medical Science, Vakilabad Blvd, Mashhad, Iran \\ Tel: 0098- 51- 38829504; Email: Farzaneh.Ahrari@Gmail. com,Ahrarif@mums.ac.ir Alireza Ghasemzadeh, Email: Ghasemzadeha991@ mums.ac.irmums.ac.ir}

Received: September 20, 2020 Accepted: January 6, 2021 Published online July 19, 2021

\begin{abstract}
Introduction: This study evaluated the effectiveness of an 810-nm diode laser as an adjunct to scaling and root planning (SRP) in improving periodontal parameters in patients with chronic periodontitis. Methods: This randomized clinical trial consisted of 36 patients (16 females and 20 males) with chronic periodontitis and pocket depths of $4-6 \mathrm{~mm}$. The quadrants were randomly divided into two sides; one side of each patient was selected as the laser group (SRP + laser) and the other side served as the control group (SRP alone). An 810-nm diode laser was applied in the laser side to remove the outer gingival epithelium (1.5 W, CW) as well as the inner epithelium of the periodontal pockets (1 $\mathrm{W}, \mathrm{CW}$ ). The clinical parameters including bleeding on probing (BOP), probing depth (PD), plaque index $(\mathrm{PI})$, and clinical attachment level $(\mathrm{CAL})$ were measured at baseline and 6 and 18 weeks after therapy.

Results: In both groups, there was a significant improvement in BOP, PD, PI and CAL over the course of the experiment $(P<0.001)$. Significantly lower BOP was found in the SRP + laser group than the SRP alone group after 6 and 18 weeks of intervention $(P<0.05)$. The difference in other parameters was not significant between the two groups, neither at 6 nor at 18 weeks after the treatment $(P>0.05)$. Conclusion: Within the limitations of this study, the association of the diode laser with standard nonsurgical periodontal therapy (SRP) provided minimal additional benefits for patients with moderate chronic periodontitis.

Keywords: Laser; Periodontal disease; Chronic periodontitis; Periodontal index; Diode
\end{abstract}

\section{Introduction}

Periodontal disease is a chronic inflammatory condition that results from the build-up of microbial plaque on the teeth and can lead to gingivitis, gingival recession, formation of periodontal pockets, vertical and/or horizontal alveolar bone resorption, and eventually, mobility and loss of the teeth. ${ }^{1}$ In the United States, chronic periodontitis affects about $47 \%$ of the subjects aged over 30 years. $^{2}$ In the treatment of periodontal disease, the goal is to stop the plaque-associated inflammation by removing supra- and subgingival microorganisms, especially anaerobic gram-negative bacilli. For this reason, the first phase of periodontal therapy consists of oral hygiene instructions as well as scaling and root planning (SRP) to remove the bacterial infection manually or through using an ultrasonic device.
Although SRP is the fundamental of periodontal therapy, it cannot completely remove pathogenic microorganisms and their endotoxins, especially in deep periodontal pockets and furcation sites. ${ }^{3,4}$ It has been proposed that the use of complementary therapies such as antibiotics, chlorhexidine or lasers in addition to SRP can provide beneficial effects on the removal of microbial plaque and reducing the number of periodontal pathogens..$^{5-7}$

The use of lasers as an adjunct to conventional periodontal treatment (SRP) has been suggested due to their proven benefits including bactericidal, hemostatic, and anti-inflammatory effects as well as their ability to accelerate the wound healing process. ${ }^{8-13}$ Amongst the different lasers introduced for dental applications, the diode laser is the most popular for soft tissue surgery as it is a small, light, durable and relatively inexpensive device. ${ }^{14-17}$

Please cite this article as follows: Mokhtari MR, Ahrari F, Dokouhaki S, Fallahrastegar A, Ghasemzadeh A. Effectiveness of an 810-nm diode laser in addition to non-surgical periodontal therapy in patients with chronic periodontitis: a randomized single-blind clinical trial. J Lasers Med Sci. 2021;12:e37. doi:10.34172/jlms.2021.37 
Furthermore, the diode laser has high absorption in pigmented materials such as melanin and hemoglobin. This property is very important in the treatment process, considering that most bacteria causing periodontal problems are pigmented. Other non-pigmented bacteria such as Actinobacillus actinomycetemcomitans are generally sensitive to heat and are removed after contact with the hot optical fiber of the laser. ${ }^{18-20}$

There are inconsistent results in the literature regarding the clinical benefits of laser therapy as an adjunct to conventional SRP in improving periodontal health in patients with chronic periodontitis. A number of investigators such as Moritz et al, ${ }^{11}$ Saafan et $\mathrm{al}^{21}{ }^{21}$ and Saglam et $\mathrm{al}^{22}$ concluded that combining diode laser radiation with mechanical treatment (SRP) can reduce the number of periodontal active pathogens and improve the attachment gain and clinical indices in subjects with periodontal problems. In contrast, others have shown that the association of laser and SRP provides no additional superiority over SRP alone in periodontally compromised patients. ${ }^{23-26}$

This study was conducted to investigate the effectiveness of applying an $810-\mathrm{nm}$ diode laser as an adjunct to conventional periodontal therapy (SRP) in improving clinical parameters in patients with chronic periodontitis by comparing the results with cases that underwent SRP alone.

\section{Material and Methods \\ Patients}

This study was a parallel-group, single-blind, randomized controlled trial with equally assigned quadrants between the right and left sides. The sample comprised 36 adult patients including 16 females and 20 males with the mean age of $41.4 \pm 8.4$ years, who were referred to the Department of Periodontology at the School of Dentistry of Mashhad University of Medical Sciences. The sample size was calculated according to the data obtained from a previous study, ${ }^{3}$ using an alpha significance level of 0.05 and a beta of 0.2 . The sample size was then increased by about $10 \%$ to counteract the possible dropout of the participants during the period of the experiment. The inclusion criteria consisted of patients older than 18 years with chronic periodontitis showing pocket depths of 4-6 mm in at least four anterior or posterior teeth at each of the right and left quadrants of the upper/lower jaw. Patients who received periodontal treatment over the 12 months preceding the study, those who consumed antibiotics or anti-inflammatory drugs within the last three months, and patients with systemic disorders that could affect the results and prognosis of periodontal treatment (such as diabetes mellitus) were excluded from the trial. The excluded were also pregnant, smokers or alcohol drinkers, patients having partial dentures, as well as those with Grade III dental mobility. The protocol of the study was approved by the ethics committee of Mashhad University of Medical Sciences and was recorded in the Iranian Registry of Clinical Trials with IRCT number IRCT20091118002736N3. The treatment protocol was explained to the patients, and they signed informed consent forms before entering the study. The experimentation was conducted in accordance with the Declaration of Helsinki.

\section{Treatment Protocols}

After filling complete medical and periodontal records, all patients received initial periodontal therapy consisting of full-mouth supragingival and subgingival scaling using hand instruments and a sonic device (Bonart Co., LTD. New Taipei City, Taiwan) with the frequency of $6000 \mathrm{~Hz}$ and constant water irrigation. The patients were trained about oral hygiene measures and were asked to brush with a modified bath technique and rinse twice daily with chlorhexidine mouthwash for two weeks after the initial periodontal therapy. The next appointment was scheduled two weeks later and SRP was repeated with hand instruments to remove the remaining plaque and calculus, followed by prophylaxis with a rubber cup and prophylactic paste. After thorough oral rinsing, the quadrants were divided into two sides (left and right); one side was randomly selected as the control group (SRP), and the other side as the laser group (SRP + laser). All teeth in the quadrant that had pocket depth between 4 and $6 \mathrm{~mm}$ were sampled. The test and control sides were determined randomly using a table of random numbers. The random allocation was concealed in sealed envelopes and was kept by an independent subject who was not involved in the study process.

The periodontal pockets of the teeth on the laser side were irradiated with a gallium aluminum arsenide (GaAlAs) diode laser (810 nm, ARC Laser $\mathrm{GmbH}$, Nürnberg, Germany) along with a 300 microns optical fiber delivery system. The laser therapist anesthetized the treatment area by injecting $1.8 \mathrm{ml}$ of $2 \%$ lidocaine with 1:100 000 epinephrine along the buccal and lingual/palatal area of the teeth per treated quadrant. Initially, the laser was set at $1.5 \mathrm{~W}$ in the continuous wave (CW) mode. After initiating the fiber tip on a bottle cork, deepithelialization of the gingiva was performed throughout the quadrant surface, removing the outer epithelial layer in a zone of attached gingiva measuring about $2 \mathrm{~mm}$ in width from the margin of free gingiva. The power was then reduced to $1 \mathrm{~W}$ for sulcular debridement in order to remove the junctional epithelium. For this purpose, the tip was inserted within the periodontal pocket and kept at the distance of $1 \mathrm{~mm}$ from the bottom, parallel to the long axis of the tooth. The tip was then gradually directed to the coronal area with a short sweeping motion towards the mesial and distal sides.

This procedure was performed at six points around the 
tooth, including mesiobuccal, midbuccal, distobuccal, mesiolingual, midlingual, and distolingual sites. The residues of sulcular epithelium and infected granulation tissues were cleaned from the laser tip frequently using sterile gauze soaked in normal saline solution. Eventually, the patient rinsed his/her mouth with saline and was asked to refrain from eating sour and spicy foods for 24 hours to avoid pain and irritation. The patients were also asked to wash their mouth with saline over two days after the treatment. From the third day, they were allowed to brush their teeth with a soft toothbrush and toothpaste.

\section{Clinical Evaluation}

The clinical measurements were contemplated before the treatment (baseline) and over 4 months after therapy. Four clinical periodontal parameters were measured by a trained and calibrated examiner using a graduated periodontal probe. The subject who assessed the outcomes was not aware of the test and control sides. The periodontal indices evaluated in this study were as follows:

Bleeding on probing (BOP): To determine BOP, the periodontal probe was inserted approximately $2 \mathrm{~mm}$ into the gingival sulcus at the buccal and lingual sides of each tooth. The probe was gradually slid from the distal to the mesial interproximal area. The presence of gingival bleeding was recorded at the distobuccal, midbuccal and mesiobuccal sites as well as the middle area of the lingual surface. Overall, BOP was registered as a yes/no response at 4 sites of every tooth. Finally, the number of sites where bleeding was present was divided by the total number of evaluated sites in the mouth and the result was multiplied by 100 to express the bleeding index as a percentage.

Probing depth (PD): PD is defined as the distance in $\mathrm{mm}$ between the free gingival margin and the bottom of the periodontal pocket. A periodontal probe was used to measure the depth of a sulcus or periodontal pocket. The periodontal probe was placed in the gingival sulcus and entered as far as possible into the pocket parallel to the longitudinal axis of the tooth. The pocket depth was recorded in millimeters at four points of each dental element; three points at the mesial, middle, and distal of the buccal surface and one point at the middle of the lingual/palatal surface.

Plaque index (PI): To determine PI, plaque detection tablets were used and all dental surfaces (except the occlusal surface) were examined for the presence of colored deposits at the dentogingival margin. The PI was calculated by dividing the number of plaque containing surfaces by the total number of available surfaces (four surfaces per tooth) and multiplying the result by 100 .

Clinical attachment level (CAL): CAL is determined by measuring the distance in millimeters from the cementoenamel junction (CEJ) to the bottom of the periodontal pocket using a periodontal probe. This criterion is a manifestation of the clinical adhesion loss of the periodontal fibers. CAL was measured at the same sites described for PD.

\section{Follow-up Intervals}

The patients were recalled at 6 and 18 weeks after the intervention and the clinical indices were measured again. Oral hygiene instructions were repeated at each recall interval.

\section{Statistical Analyses}

Data were collected and analyzed with SPSS statistical software package, ver. 16.0 (SPSS Inc., Chicago, IL, USA). The values of clinical parameters at different time points were compared using Friedman and Wilcoxon signed rank tests. Between group comparisons for BOP, PD, $\mathrm{PI}$ and CAL were made by Mann-Whitney $\mathrm{U}$ test. The significance level was set at $P<0.05$.

\section{Results}

All the patients were followed up until the end of the study. Tables 1 to 4 present the means and standard deviations (SD) of the clinical parameters in the "SRP alone" and "SRP plus laser" groups at baseline and at 6 and 18 weeks later. In both groups, there was a significant decrease in BOP, PD, PI and the CAL over the period of the experiment $(P<0.001$; Tables $1-4)$.

Table 1. The Mean and Standard Deviation of BOP in SRP and SRP + Laser Groups at Baseline (T0) and 6 (T1) and 18 (T2) Weeks After Therapy

\begin{tabular}{|c|c|c|c|c|c|}
\hline & \multicolumn{2}{|c|}{ SRP } & \multicolumn{2}{|c|}{ SRP + Laser } & \multirow{2}{*}{$P$ Value } \\
\hline & Mean & SD & Mean & SD & \\
\hline Before treatment (T0) & 65.27 & 28.65 & 65.3 & 28.11 & 0.95 \\
\hline 6 weeks after treatment (T1) & 31.61 & 19.2 & 22.5 & 11.71 & $0.01 *$ \\
\hline 18 weeks after treatment (T2) & 18.41 & 12.17 & 12.11 & 9.05 & $0.02 *$ \\
\hline$P$ value & \multicolumn{2}{|c|}{$<0.001^{*}$} & \multicolumn{2}{|c|}{$<0.001^{*}$} & \\
\hline Pairwise comparisons & \multicolumn{2}{|c|}{$\begin{array}{ll}\text { T0-T1 } & P<0.001^{*} \\
\text { T0-T2 } & P<0.001 * \\
\text { T1-T2 } & P<0.001 *\end{array}$} & \multicolumn{2}{|c|}{$\begin{array}{l}\text { T0-T1 } P<0.001^{*} \\
\text { T0-T2 } P<0.001^{*} \\
\text { T1-T2 } P<0.001^{*}\end{array}$} & \\
\hline
\end{tabular}

*indicates statistically significant difference at $P<0.05$. 
Table 2. The Mean and Standard Deviation of PD (mm) in SRP and SRP + Laser Groups at Baseline (T0) and at 6 (T1) and 18 (T2) Weeks After Therapy

\begin{tabular}{|c|c|c|c|c|c|}
\hline & \multicolumn{2}{|c|}{ SRP } & \multicolumn{2}{|c|}{ SRP + Laser } & \multirow{2}{*}{$P$ Value } \\
\hline & Mean & SD & Mean & SD & \\
\hline Before treatment (T0) & 4.88 & 0.6 & 4.71 & 0.55 & 0.2 \\
\hline 6 weeks after treatment (T1) & 4.34 & 0.83 & 4.1 & 0.84 & 0.22 \\
\hline 18 weeks after treatment (T2) & 4.23 & 0.84 & 3.94 & 0.85 & 0.2 \\
\hline$P$ value & \multicolumn{2}{|c|}{$<0.001^{*}$} & \multicolumn{2}{|c|}{$<0.001^{*}$} & \\
\hline Pairwise comparisons & \multicolumn{2}{|c|}{$\begin{array}{l}\text { T0-T1 } P<0.001 * \\
\text { T0-T2 } P<0.001 * \\
\text { T1-T2 } P=0.003^{*}\end{array}$} & \multicolumn{2}{|c|}{$\begin{array}{l}\text { T0-T1 } P<0.001 * \\
\text { T0-T2 } P<0.001 * \\
\text { T1-T2 } P<0.001^{*}\end{array}$} & \\
\hline
\end{tabular}

*indicates statistically significant difference at $P<0.05$.

Table 3. The Mean and Standard Deviation of PI in SRP and SRP + Laser Groups at Baseline (T0) and at 6 (T1) and 18 (T2) Weeks After Therapy

\begin{tabular}{|c|c|c|c|c|c|}
\hline & \multicolumn{2}{|c|}{ SRP } & \multicolumn{2}{|c|}{ SRP + laser } & \multirow{2}{*}{$P$ Value } \\
\hline & Mean & SD & Mean & SD & \\
\hline Before treatment (T0) & 71.52 & 23.92 & 70.91 & 23.94 & 0.94 \\
\hline 6 weeks after treatment (T1) & 46.86 & 22.4 & 46.83 & 21.95 & 0.94 \\
\hline 18 weeks after treatment $(\mathrm{T} 2)$ & 40.25 & 21.21 & 38.8 & 21.14 & 0.79 \\
\hline$P$ value & \multicolumn{2}{|c|}{$<0.001^{*}$} & \multicolumn{2}{|c|}{$<0.001^{*}$} & \\
\hline Pairwise comparisons & \multicolumn{2}{|c|}{$\begin{array}{l}\text { T0-T1 } P<0.001^{*} \\
\text { T0-T2 } P<0.001^{*} \\
\text { T1-T2 } P=0.001^{*}\end{array}$} & \multicolumn{2}{|c|}{$\begin{array}{l}\text { T0-T1 } P<0.001^{*} \\
\text { T0-T2 } P<0.001^{*} \\
\text { T1-T2 } P<0.001^{*}\end{array}$} & \\
\hline
\end{tabular}

*indicates statistically significant difference at $P<0.05$.

Table 4. The Mean and Standard Deviation of CAL (mm) in SRP and SRP + Laser Groups at Baseline (T0) and at 6 (T1) and 18 (T2) Weeks After Therapy

\begin{tabular}{|c|c|c|c|c|c|}
\hline & \multicolumn{2}{|c|}{ SRP } & \multicolumn{2}{|c|}{ SRP + Laser } & \multirow{2}{*}{$P$ Value } \\
\hline & Mean & SD & Mean & SD & \\
\hline Before treatment (T0) & 5.15 & 1.41 & 4.98 & 1.36 & 1.0 \\
\hline 6 weeks after treatment (T1) & 4.59 & 1.56 & 4.37 & 1.65 & 0.8 \\
\hline 18 weeks after treatment (T2) & 4.42 & 1.41 & 4.13 & 1.42 & 1.0 \\
\hline$P$ value & \multicolumn{2}{|c|}{$<0.001^{*}$} & \multicolumn{2}{|c|}{$<0.001^{*}$} & \\
\hline Pairwise comparisons & \multicolumn{2}{|c|}{$\begin{array}{l}\text { T0-T1 } P=0.021^{*} \\
\text { T0-T2 } P=0.008^{*} \\
\text { T1-T2 } P=0.015^{*}\end{array}$} & \multicolumn{2}{|c|}{$\begin{array}{c}\text { T0-T1 } P=0.01 * \\
\text { T0-T2 } P=0.008^{*} \\
\text { T1-T2 } P=0.04 *\end{array}$} & \\
\hline
\end{tabular}

*indicates statistically significant difference at $P<0.05$.

At baseline, there was no significant difference in any of the clinical parameters between the two groups $(P>0.05)$. Significant differences between the laser and control groups were found in terms of BOP after 6 and 18 weeks of intervention $(P<0.05$; Table 1$)$. Regarding the probing depth, plaque index, and clinical attachment level, the difference between the two groups was not significant neither at 6 nor at 18 weeks after the treatment $(P>0.05$; Tables 2-4).

\section{Discussion}

This study evaluated the effectiveness of an 810-nm diode laser in addition to conventional SRP in improving periodontal parameters in patients with chronic periodontitis. The diode laser was applied in this study for several purposes including the removal of the outer gingival epithelium, eliminating the inner epithelial layer of the pocket (subgingival curettage), and reducing the population of pathogenic bacteria. Initially, the outer gingiva was deepithelialized in a zone around $2 \mathrm{~mm}$ in width from the margin of free gingiva at the power setting of $1.5 \mathrm{~W}$. This was done to prevent the growth of the outer epithelial layer into the gingival sulcus. Afterwards, the power output was reduced to $1 \mathrm{~W}$ and the laser optical fiber was entered $1 \mathrm{~mm}$ less than the pocket depth into the gingival sulcus. Through sweeping motion to prevent thermal side effects, the infected sulcular epithelium and granulation tissues were gently removed. It has been 
revealed that the regeneration rate of the epithelium is faster than that of fibroblasts and osteoblasts. ${ }^{27}$ Therefore, the outer and inner gingival epithelium was removed during the laser treatment to facilitate the fibroblast activity and enhance regeneration through connective tissue attachment to the root surface. Laser treatment of the pocket is also assumed to improve the repair process by eradicating the subgingival pathogenic bacteria.

Bleeding on probing (BOP) is a manifestation of inflammation. It is a very popular and commonly-used criterion to diagnose gingivitis and periodontitis and identify the sites at risk for periodontal breakdown. In the present study, a significant decrease in the BOP index was observed in both the control and the laser groups. For the SRP group, the reduction in BOP index was 52\% and $72 \%$ after 6 and 18 weeks of treatment respectively, whereas it was $66 \%$ and $81 \%$ for the SRP + laser group. The between-group comparison revealed that BOP was significantly lower in the quadrants that underwent SRP + laser treatment, as compared to those that received SRP alone. This improvement may be attributed to decreased inflammation due to the eradication of periodontal pathogens and the elimination of the infected sulcular epithelium and granulation tissue from the periodontal pockets.

In the present study, both the laser and control groups showed a significant decrease in the pocket depth (PD) and plaque index (PI) following the treatment. However, the difference between the two groups was small and not statistically significant. The pocket depth is generally between 1 and $3 \mathrm{~mm}$ in a healthy periodontium. Pockets deeper than $4 \mathrm{~mm}$ indicate periodontitis and cannot be cleaned well. The PI is employed to estimate the status of oral hygiene by measuring the dental plaque located around the gingival margin. The outcomes of this study revealed that the adjunctive use of a diode laser with mechanical instrumentation of the root surface (SRP) has no remarkable advantages in terms of the probing depth and PI in subjects with chronic periodontitis.

The clinical attachment level (or loss, CAL) is another measurement of the extent of the periodontal support and refers to the pathological detachment of connective tissue from the cementum surface. CAL is a more accurate indicator of the periodontal support around a tooth than the probing depth alone. CAL is measured from an unchangeable point on the tooth surface, the CEJ. In the present study, CAL showed a small but significant improvement after the treatment in both the SRP + laser and SRP alone groups. However, no significant difference was observed between the two groups at any of the assessment intervals.

The overall outcomes of this study indicated that the traditional mechanical procedure of SRP either alone or combined with diode laser radiation provided a significant improvement in clinical parameters (BOP,
PD, PI and CAL) during the observation period of this experiment. The only superiority of laser addition to SRP was significantly lower BOP after 6 and 18 weeks of treatment compared to the SRP alone group. Therefore, laser radiation of the periodontal pockets could have some clinical outcomes in improving clinical parameters in patients with chronic periodontitis, but the benefit is small and laser treatment should be considered only as an adjunct to non-surgical periodontal therapy.

The insufficient success in the use of diode laser for improving clinical parameters could be attributed to the chosen laser parameters as well as to the short duration of the follow-up period of this experiment. It is also possible that laser-exposed tooth surfaces experience some irregularities which is better to smooth out by second SRP after laser radiation; this procedure was not performed in the present investigation. Frequent subgingival laser applications over 1 week after SRP have also been suggested by some authors, but in this study, the laser was applied only once.

The results of this study comply with a number of investigations that reported only a slight improvement in clinical periodontal parameters upon employing lasers together with conventional mechanical debridement of the root surfaces. ${ }^{3,25,28-30}$ Zare et $\mathrm{al}^{29}$ investigated the effectiveness of a diode laser $(980 \mathrm{~nm})$ in nonsurgical periodontal therapy and found improvement in periodontal indices in both test (SRP + laser) and control (SRP) groups, with significant superiority of the test group in just BOP index. Other studies concluded that the application of a diode laser as an adjunct to conventional periodontal therapy provides no additional benefits in terms of clinical or microbiologic indices for patients with chronic periodontitis. ${ }^{1,23,24,31-35}$ Balasubramaniam et $\mathrm{al}^{1}$ exhibited that employing a diode laser as an adjunct to SRP provides no advantages compared to the use of SRP alone regarding clinical parameters and reactive oxygen metabolites in patients with chronic periodontitis.

In contrast to the outcomes of this study, there are some clinical studies that supported significant advantages for the diode laser-mediated periodontal therapy., ${ }^{2,420,22,36-40}$ Üstün et $\mathrm{al}^{4}$ displayed that the application of the diode laser as an adjunctive periodontal treatment performed significantly better than SRP alone in terms of both clinical and biochemical parameters. The discrepancy observed between the results of this study and those of previous studies may be attributed to the differences in wavelength, power, frequency and duration of laser irradiation, as well as to the variations in the study design.

One of the limitations of this study was the short duration of the follow-up period. Additional randomized, clinical trials with larger sample sizes and longer followups are warranted to better investigate any possible benefits of adding laser therapy to conventional non-surgical periodontal treatment. Alterations in laser parameters 
and modes of application are also recommended to attain better clinical results.

\section{Conclusion}

Under the conditions of this study, the association of diode laser irradiation with conventional SRP significantly improved the BOP index at 6 and 18 weeks after the treatment, when compared to SRP alone. However, there was no significant difference in other clinical parameters between the test ad control sides. Therefore, the use of an $810-\mathrm{nm}$ diode laser as an adjunct to the traditional nonsurgical periodontal therapy provides minimal clinical benefits for patients with moderate chronic periodontitis.

\section{Ethical Considerations}

The study protocol was approved by the ethics committee of Mashhad University of Medical Sciences and was registered in the Iranian Registry of Clinical Trials (identifier: IRCT20091118002736N3)

\section{Conflict of Interests}

The authors declare that they have no conflict of interest.

\section{Acknowledgements}

The authors would like to thank the vice chancellor for research of Mashhad University of Medical Sciences for the financial support of this project (grant number 940131). The results presented in this work have been taken from a DDS student thesis.

\section{References}

1. Balasubramaniam AS, Thomas LJ, Ramakrishnanan T, Ambalavanan N. Short-term effects of nonsurgical periodontal treatment with and without use of diode laser $(980 \mathrm{~nm})$ on serum levels of reactive oxygen metabolites and clinical periodontal parameters in patients with chronic periodontitis: a randomized controlled trial. Quintessence Int. 2014;45(3):193-201. doi: 10.3290/j.qi.a31206

2. Crispino A, Figliuzzi MM, Iovane C, Del Giudice T, Lomanno S, Pacifico D, et al. Effectiveness of a diode laser in addition to non-surgical periodontal therapy: study of intervention. Ann Stomatol (Roma). 2015;6(1):15-20.

3. Dukic W, Bago I, Aurer A, Roguljic M. Clinical effectiveness of diode laser therapy as an adjunct to nonsurgical periodontal treatment: a randomized clinical study. J Periodontol. 2013;84(8):1111-7. doi: 10.1902/ jop. 2012.110708

4. Üstün K, Erciyas K, Sezer U, Senyurt SZ, Gundogar H, Ustun $\mathrm{O}$, et al. Clinical and biochemical effects of 810 $\mathrm{nm}$ diode laser as an adjunct to periodontal therapy: a randomized split-mouth clinical trial. Photomed Laser Surg. 2014;32(2):61-6. doi: 10.1089/pho.2013.3506

5. Smiley CJ, Tracy SL, Abt E, Michalowicz BS, John MT, Gunsolley J, et al. Systematic review and meta-analysis on the nonsurgical treatment of chronic periodontitis by means of scaling and root planing with or without adjuncts. J Am Dent Assoc. 2015;146(7):508-24. e5. doi: 10.1016/j. adaj.2015.01.028.

6. Faveri M, Gursky LC, Feres M, Shibli JA, Salvador SL, De
Figueiredo LC. Scaling and root planing and chlorhexidine mouthrinses in the treatment of chronic periodontitis: A randomized, placebo-controlled clinical trial. J Clin Periodontol. 2006;33(11):819-28.

7. Birang R, Shahaboui M, Kiani S, Shadmehr E, Naghsh N. Effect of Nonsurgical Periodontal Treatment Combined With Diode Laser or Photodynamic Therapy on Chronic Periodontitis: A Randomized Controlled Split-Mouth Clinical Trial. J Lasers Med Sci. 2015;6(3):112-9. doi: 10.15171/jlms.2015.04

8. Harris DM, Yessik M. Therapeutic ratio quantifies laser antisepsis: ablation of Porphyromonas gingivalis with dental lasers. Lasers Surg Med. 2004;35(3):206-13. doi: $10.1002 / \mathrm{lsm} .20086$

9. Jahanbin A, Ramazanzadeh B, Ahrari F, Forouzanfar A, Beidokhti M. Effectiveness of Er: YAG laser-aided fiberotomy and low-level laser therapy in alleviating relapse of rotated incisors. Am J Orthod Dentofacial Orthop. 2014;146(5):565-72. doi: 10.1016/j.ajodo.2014.07.006

10. Pakfetrat A, Falaki F, Ahrari F, Bidad S. Removal of refractory erosive-atrophic lichen planus by the $\mathrm{CO} 2$ laser. Oral Health Dent Manag. 2014;13(3):595-9.

11. Moritz A, Schoop U, Goharkhay K, Schauer P, Doertbudak $\mathrm{O}$, Wernisch J, et al. treatment of periodontal pockets with a diode laser. Lasers Surg Med. 1998;22(5):302-11.

12. Bidar M, Moushekhian S, Gharechahi M, Talati A, Ahrari F, Bojarpour M. The Effect of Low Level Laser Therapy on Direct Pulp Capping in Dogs. J Lasers Med Sci. 2016;7(3):177-83. doi: 10.15171/jlms.2016.31

13. Sardari F, Ahrari F. The effect of low-level helium-neon laser on oral wound healing. Dent Res J (Isfahan). 2016;13(1):249. doi: 10.4103/1735-3327.174693

14. Gupta S, Kumar S. Lasers in Dentistry-An Overview. Trends Biomater Artif Organs. 2011;25(3):119-23.

15. Ahrari F, Akbari M, Mohammadpour S, Forghani M. The efficacy of laser-assisted in-office bleaching and home bleaching on sound and demineralized enamel. Laser Ther. 2015;24(4):257-64. doi: 10.5978/islsm.15-OR-15

16. Ahrari F, Mohammadipour HS, Hajimomenian L, FallahRastegar A. The effect of diode laser irradiation associated with photoabsorbing agents containing remineralizing materials on microhardness, morphology and chemical structure of early enamel caries. J Clin Exp Dent. 2018;10(10): e955-e62. doi: 10.4317/jced.55059

17. Ghanbarzadeh $M$, Ahrari F, Akbari M, Hamzei H. Microhardness of demineralized enamel following home bleaching and laser-assisted in office bleaching. J Clin Exp Dent. 2015;7(3): e405-9. doi: 10.4317/jced.51705

18. Gutknecht N, Franzen R, Schippers M, Lampert F. Bactericidal effect of a $980-\mathrm{nm}$ diode laser in the root canal wall dentin of bovine teeth. J Clin Laser Med Surg. 2004;22(1):9-13. doi:10.1089/104454704773660912

19. Gutknecht N, van Gogswaardt D, Conrads G, Apel C, Schubert C, Lampert F. Diode laser radiation and its bactericidal effect in root canal wall dentin. J Clin Laser Med Surg. 2000;18(2):57-60. doi:10.1089/clm.2000.18.57

20. Kreisler M, Al Haj H, d'Hoedt B. Clinical efficacy of semiconductor laser application as an adjunct to conventional scaling and root planing. Lasers Surg Med. 2005;37(5):350-5. doi: 10.1002/lsm.20252 
21. Saafan A, El-Nahass H, Nasr A, Radwan R. Effect of low power diode laser $810 \mathrm{~nm}$ on TGF- \& 946;1 level in GCF in aggressive periodontitis. J Dent Lasers 2013;7(2):59-65. doi: 10.4103/0976-2868.124266

22. Saglam M, Kantarci A, Dundar N, Hakki SS. Clinical and biochemical effects of diode laser as an adjunct to nonsurgical treatment of chronic periodontitis: a randomized, controlled clinical trial. Lasers Med Sci. 2014;29(1):37-46. doi: 10.1007/s10103-012-1230-0

23. Euzebio Alves VT, de Andrade AK, Toaliar JM, Conde MC, Zezell DM, Cai S, et al. Clinical and microbiological evaluation of high intensity diode laser adjutant to nonsurgical periodontal treatment: a 6-month clinical trial. Clin Oral Investig. 2013;17(1):87-95. doi: 10.1007/s00784012-0703-7

24. Matthews DC. Seeing the Light--the truth about soft tissue lasers and nonsurgical periodontal therapy. J Can Dent Assoc. 2010;76: a30.

25. Caruso U, Nastri L, Piccolomini R, d'Ercole S, Mazza C, Guida L. Use of diode laser $980 \mathrm{~nm}$ as adjunctive therapy in the treatment of chronic periodontitis. A randomized controlled clinical trial. New Microbiol. 2008;31(4):513-8.

26. Polansky R, Haas M, Heschl A, Wimmer G. Clinical effectiveness of photodynamic therapy in the treatment of periodontitis. J Clin Periodontol. 2009;36(7):575-80.

27. Polimeni G, Xiropaidis AV, Wikesjö UME. Biology and principles of periodontal wound healing/regeneration. Periodontol 2000. 2006;41(1):30-47. doi: 10.1111/j.16000757.2006.00157.x

28. Shah C, Modi B, Budhiraja S, Desai K. A Short Term Comparative clinical evaluation of diode laser and hand Instruments for gingival curettage. Adv Hum Biol.2013;3(1):37-42.

29. Zare D, Haerian A, Molla R, Vaziri F. Evaluation of the effects of diode $(980 \mathrm{~nm}$ ) laser on gingival inflammation after nonsurgical periodontal therapy. J Lasers Med Sci. 2014;5(1):27-31.

30. Chambrone L, Ramos UD, Reynolds MA. Infrared lasers for the treatment of moderate to severe periodontitis: An American Academy of Periodontology best evidence review. J Periodontol. 2018;89(7):743-65. doi: 10.1902/ jop.2017.160504

31. De Micheli G, de Andrade AK, Alves VT, Seto M, Pannuti CM, Cai S. Efficacy of high intensity diode laser as an adjunct to non-surgical periodontal treatment: a randomized controlled trial. Lasers Med Sci. 2011;26(1):438. doi: 10.1007/s10103-009-0753-5

32. Lin J, Bi L, Wang L, Song Y, Ma W, Jensen S, et al. Gingival curettage study comparing a laser treatment to hand instruments. Lasers Med Sci. 2011;26(1):7-11. doi: 10.1007/ s10103-009-0732-x

33. Makhlouf M, Dahaba MM, Tuner J, Eissa SA, Harhash TA. Effect of adjunctive low level laser therapy (LLLT) on nonsurgical treatment of chronic periodontitis. Photomed Laser Surg. 2012;30(3):160-6. doi: 10.1089/pho.2011.3069

34. Meseli SE, Kuru B, Kuru L. Effects of 810-nanometer diode laser as an adjunct to mechanical periodontal treatment on clinical periodontal parameters and gingival crevicular fluid volume of residual periodontal pockets. Niger J Clin Pract 2017;20(4):427-32. doi: 10.4103/1119-3077.181382

35. Yadwad KJ, Veena HR, Patil SR, Shivaprasad BM. Diode laser therapy in the management of chronic periodontitis - A clinico-microbiological study. Interv Med Appl Sci. 2017;9(4):191-8. doi: 10.1556/1646.9.2017.38

36. Gokhale SR, Padhye AM, Byakod G, Jain SA, Padbidri V, Shivaswamy S. A comparative evaluation of the efficacy of diode laser as an adjunct to mechanical debridement versus conventional mechanical debridement in periodontal flap surgery: a clinical and microbiological study. Photomed Laser Surg. 2012;30(10):598-603. doi: 10.1089/ pho.2012.3252

37. Kamma JJ, Vasdekis VG, Romanos GE. The effect of diode laser $(980 \mathrm{~nm})$ treatment on aggressive periodontitis: evaluation of microbial and clinical parameters. Photomed Laser Surg. 2009;27(1):11-9. doi: 10.1089/pho.2007.2233

38. Ribeiro IW, Sbrana MC, Esper LA, Almeida AL. Evaluation of the effect of the GaAlAs laser on subgingival scaling and root planing. Photomed Laser Surg. 2008;26(4):387-91. doi: 10.1089/pho.2007.2152

39. Dengizek Eltas S, Gursel M. Evaluation of long-term effects of diode laser application in periodontal treatment of poorly controlled type 2 diabetic patients with chronic periodontitis. Int J Dent Hyg 2019;17(4):292-9. doi: 10.1111/idh.12384

40. Talmac AC, Calisir M. Effects of Er, Cr:YSGG and diode lasers on clinical parameters and gingival crevicular fluid IL-1beta and IL-37 levels in generalized aggressive periodontitis. Mediators Inflamm. 2019; 2019:2780794. doi: $10.1155 / 2019 / 2780794$ 